\title{
Role of population transfer under strong probe conditions in electromagnetically induced transparency
}

\author{
Kanhaiya Pandey, Dipankar Kaundilya, and Vasant Natarajan* \\ Department of Physics, Indian Institute of Science, Bangalore 560 012, INDIA
}

\begin{abstract}
We analyze theoretically the phenomenon of electromagnetically induced transparency (EIT) under conditions where the probe laser is not in the usual weak limit. We consider the effects in both three-level and four-level systems, which are either closed or open (due to losses to an external metastable level). We find that the EIT dip almost disappears in a closed three-level system but survives in an open system. In four-level systems, there is a narrow enhanced-absorption peak (EITA) at line center, which has applications as an optical clock. The peak converts to an EIT dip in a closed system, but again survives in an open system.
\end{abstract}

PACS numbers: 42.50.Gy,42.50.Nn,32.80.Qk

\section{INTRODUCTION}

The phenomenon of electromagnetically induced transparency (EIT) is generally studied in multilevel systems under conditions where a strong control laser drives one transition and thereby modifies the absorption properties of the medium for a weak probe laser on another transition. The modification happens because the control laser shifts the energy levels (due to the AC-Stark effect resulting in the formation of "dressed" states) and the subsequent quantum interference among the new pathways for probe absorption. In addition, the control laser, when it couples to a ground level, causes population redistribution among the levels due to optical pumping. The probe laser is considered weak enough that it does not play a role in either shifting the energy levels or causing population transfer. EIT has important applications in diverse fields such as lasing without inversion [1, 2], highresolution spectroscopy [3, 4], enhancement of secondorder and third-order nonlinear processes [ 5,6$]$, polarization control [7, [8] and slowing of light [9]. In many of these applications, particularly when studying nonlinear processes, the weak-probe assumption is not valid. There are also recent proposals 10 12 to use control lasers in a four-level system to drive an optical clock transition. The probe laser is on a weakly-allowed intercombination line. Our experiments using a similar transition in $\mathrm{Yb}$ have shown that the probe laser must be operated near saturation intensity to get good signal-to-noise ratio from the weak transition.

We thus see that it is important to understand the role of a strong probe laser in EIT experiments. There have been two previous studies using a strong probe laser in a ladder-type system in $\mathrm{Rb}$ [13, 14]. This system can be considered closed since there are no decay pathways out of the three levels under consideration. However, in atoms such as $\mathrm{Sr}$ and $\mathrm{Yb}$, the presence of low-lying

*Electronic address: vasant@physics.iisc.ernet.in URL: www.physics.iisc.ernet.in/ vasant

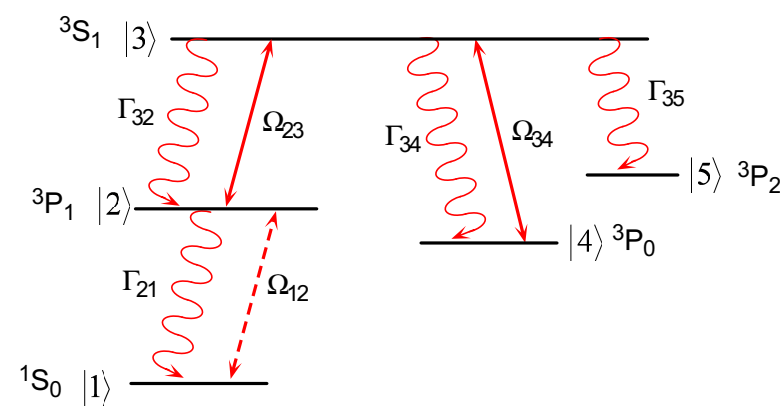

FIG. 1: Multilevel system under consideration. Shown alongside each level is the atomic state that can be used to realize this level scheme in $\mathrm{Yb}$.

metastable states makes them open or lossy. We therefore study the modification to probe absorption due to a strong probe laser in both closed and open systems. As we will see, the main effect of the strong probe is in causing additional population transfer between the levels. We also consider four-level systems due to their importance in optical-clock proposals.

\section{DENSITY-MATRIX ANALYSIS}

Consider the multi-level system shown in Fig. 1, Levels $|4\rangle$ and $|5\rangle$ are metastable levels, so that atoms decaying to these levels are lost unless they are repumped. The probe laser is on the $|1\rangle \leftrightarrow|2\rangle$ transition, while there are two control lasers, on the $|2\rangle \leftrightarrow|3\rangle$ transition and the $|3\rangle \leftrightarrow|4\rangle$ transition, respectively. The Rabi frequency of a laser driving the $|i\rangle \leftrightarrow|j\rangle$ transition is $\Omega_{i j}$ and its detuning from resonance is $\Delta_{i j}$. The corresponding decay rate from the upper level $|j\rangle$ to the lower level $|i\rangle$ is $\Gamma_{j i}$. This level scheme can be made three- or four-level, and open or closed, by choosing suitable parameters to be 0 . For example, if $\Omega_{34}$ is 0 , it becomes a three-level ladder system. Simultaneously, if $\Gamma_{34}$ and $\Gamma_{35}$ are 0 , the system becomes closed, and open otherwise. The general four-level system is again closed if $\Gamma_{35}$ is 0 , and open otherwise. 
The time evolution of the system in the rotating-wave approximation is given by the following set of densitymatrix equations. As usual, $\rho_{i i}$ gives the population in level $|i\rangle$, while $\rho_{i j}$ gives the coherence between levels $|i\rangle$ and $|j\rangle$.

$$
\begin{aligned}
& \rho_{11}=\Gamma_{21} \rho_{22}+\frac{i}{2}\left[\Omega_{12} \rho_{21}-\Omega_{12}^{*} \rho_{12}\right], \\
& \rho_{22}=-\Gamma_{21} \rho_{22}+\Gamma_{32} \rho_{33}+\frac{i}{2}\left[\Omega_{12}^{*} \rho_{12}-\Omega_{12} \rho_{21}\right] \\
& +\frac{i}{2}\left[\Omega_{23}^{*} \rho_{32}-\Omega_{23} \rho_{23}\right] \text {, } \\
& \rho_{33}=-\Gamma_{32} \rho_{33}-\Gamma_{34} \rho_{33}-\Gamma_{35} \rho_{33} \\
& +\frac{i}{2}\left[\Omega_{23}^{*} \rho_{23}-\Omega_{23} \rho_{32}\right]+\frac{i}{2}\left[\Omega_{34} \rho_{43}-\Omega_{34}^{*} \rho_{34}\right], \\
& \rho_{44}=\Gamma_{34} \rho_{33}-\Gamma_{41} \rho_{44}+\frac{i}{2}\left[\Omega_{34}^{*} \rho_{34}-\Omega_{34} \rho_{43}\right] \text {, } \\
& \rho_{12}=-\left[\frac{\Gamma_{21}}{2}+i \Delta_{12}\right] \rho_{12}+\frac{i}{2}\left[\Omega_{12}\left(\rho_{22}-\rho_{11}\right)\right] \\
& -\frac{i}{2}\left[\Omega_{23}^{*} \rho_{13}\right] \text {, } \\
& \rho_{13}=-\left[\frac{\Gamma_{32}}{2}+\frac{\Gamma_{34}}{2}+\frac{\Gamma_{35}}{2}+i\left(\Delta_{12}+\Delta_{23}\right)\right] \rho_{13} \\
& +\frac{i}{2}\left[\Omega_{12} \rho_{23}-\Omega_{23} \rho_{12}-\Omega_{34}^{*} \rho_{14}\right], \\
& \rho_{14}=-\left[\frac{\Gamma_{41}}{2}+i\left(\Delta_{12}+\Delta_{23}-\Delta_{34}\right)\right] \rho_{14} \\
& +\frac{i}{2}\left[\Omega_{12} \rho_{24}-\Omega_{34} \rho_{13}\right] \text {, } \\
& \rho_{23}=-\left[\frac{\Gamma_{32}}{2}+\frac{\Gamma_{34}}{2}+\frac{\Gamma_{35}}{2}+\frac{\Gamma_{21}}{2}+i \Delta_{23}\right] \rho_{23} \\
& +\frac{i}{2}\left[\Omega_{12} \rho_{13}+\Omega_{23}\left(\rho_{33}-\rho_{22}\right)-\Omega_{34}^{*} \rho_{24}\right], \\
& \rho_{24}=-\left[\frac{\Gamma_{21}}{2}+\frac{\Gamma_{41}}{2}+i\left(\Delta_{23}-\Delta_{34}\right)\right] \rho_{24} \\
& +\frac{i}{2}\left[\Omega_{12} \rho_{14}+\Omega_{23} \rho_{34}-\Omega_{34} \rho_{23}\right], \\
& \rho_{34}=-\left[\frac{\Gamma_{32}}{2}+\frac{\Gamma_{34}}{2}+\frac{\Gamma_{35}}{2}+\frac{\Gamma_{41}}{2}-i \Delta_{34}\right] \rho_{34} \\
& +\frac{i}{2}\left[\Omega_{23}^{*} \rho_{24}+\Omega_{34}\left(\rho_{44}-\rho_{33}\right)\right] \text {. }
\end{aligned}
$$

The usual method of solving these equations in the weak probe limit is to look for a steady-state solution to first order in the probe Rabi frequency. Since we want to see the modification due to a strong probe laser, we solve these equations numerically with arbitrary values of the Rabi frequency. The other parameters are taken from the relevant level scheme in Yb, i.e., $\Gamma_{21}=2 \pi \times 0.18$ $\mathrm{MHz}, \Gamma_{32}=2 \pi \times 5.89 \mathrm{MHz}, \Gamma_{34}=2 \pi \times 1.54 \mathrm{MHz}$, and $\Gamma_{35}=2 \pi \times 4.29 \mathrm{MHz}[15]$. The metastable state $|4\rangle$ in $\mathrm{Yb}$ has a lifetime of order months, hence the decay rate $\Gamma_{41}$ is taken to be 0 .

Of course, for open systems, there is no true steady state, since all the population will be lost to the

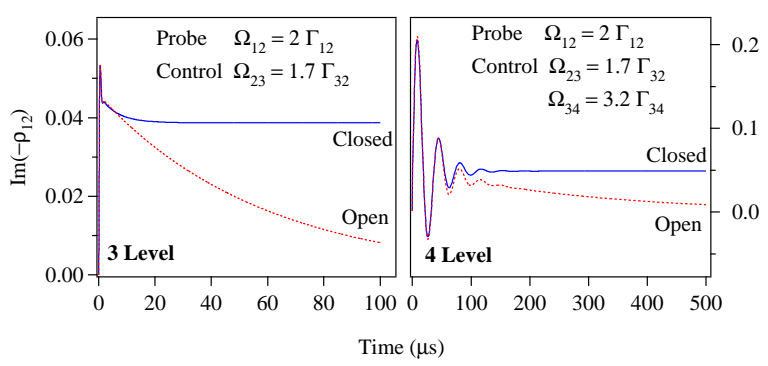

FIG. 2: Time evolution of $\operatorname{Im}\left(-\rho_{12}\right)$ for open and closed systems. For open systems, there is no steady state and the amplitude continues to decay.

metastable levels as $t \rightarrow \infty$. In Fig. 2, we show the time evolution of $\operatorname{Im}\left(-\rho_{12}\right)$ under typical strong probe conditions for both open and closed systems. In the three-level case, the transients for a closed system last a few microseconds and then stabilize to the steady state value. In an open system, the transients last for the same time, after which the amplitude continues to decay but without any no structure. Hence our results are given after $100 \mu \mathrm{s}$. For four-level systems, the transients in both systems last for almost $200 \mu \mathrm{s}$. Therefore, the results are given after $500 \mu \mathrm{s}$.

\section{RESULTS IN A THREE-LEVEL LADDER SYSTEM}

We first consider the effect of a strong probe in a threelevel ladder system. As explained earlier, we realize this system by setting $\Omega_{34}=0$. We compare the effect of the probe power in both a closed system (by setting $\Gamma_{34}=$ $\Gamma_{35}=0$ ) and an open system (with just $\Omega_{34}=0$ ).

In the density-matrix analysis, probe absorption is proportional to $\operatorname{Im}\left(-\rho_{12}\right)$, where $\rho_{12}$ is the induced absorption on the $|1\rangle \leftrightarrow|2\rangle$ transition coupled by the probe laser. In the weak-probe limit, we can assume that all the population is in the ground level, i.e., $\rho_{11}=1$, and $\rho_{22}=\rho_{33}=0$. This assumption breaks down when the probe laser is strong. If we do a series expansion in probe Rabi frequency, the solution to the density-matrix equations yields,

$$
\rho_{12}=-\frac{\frac{i}{2} \frac{\Omega_{12}}{\gamma_{12}}[1+A]-\frac{i}{8} \frac{\Omega_{23}^{2} \Omega_{12}}{\gamma_{12} \gamma_{13} \gamma_{23}}[A][1+B]}{1+\frac{\Omega_{23}^{2}}{4 \gamma_{12} \gamma_{13}}[1+B]},
$$

where

$$
\begin{aligned}
& \gamma_{12}=\Gamma_{21} / 2+i \Delta_{12}, \\
& \gamma_{13}=\left(\Gamma_{32}+\Gamma_{34}+\Gamma_{35}\right) / 2+i\left(\Delta_{12}+\Delta_{23}\right), \\
& \gamma_{23}=\left(\Gamma_{21}+\Gamma_{32}+\Gamma_{34}+\Gamma_{35}\right) / 2+i \Delta_{23},
\end{aligned}
$$




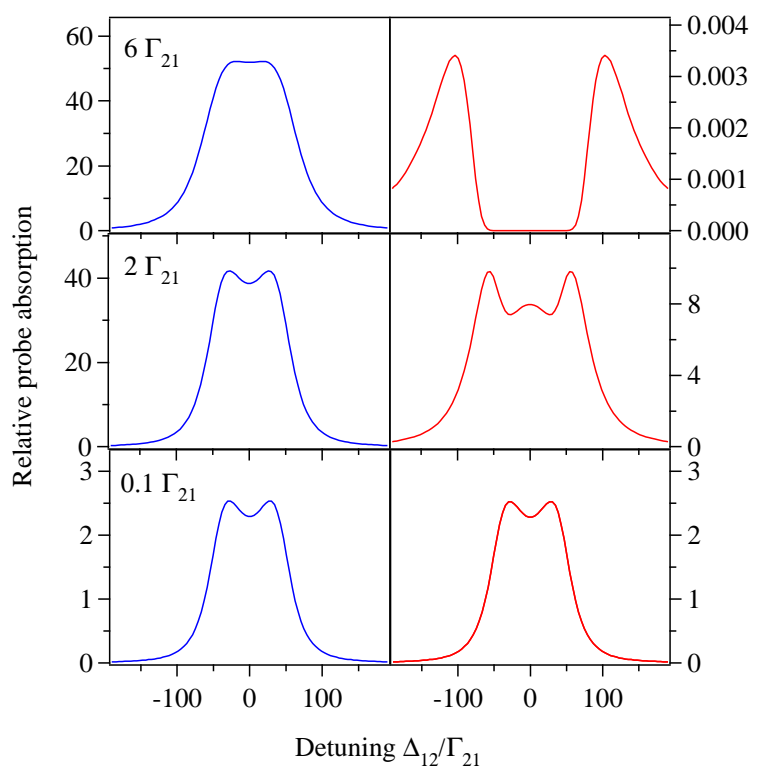

FIG. 3: Relative probe absorption [defined as $\operatorname{Im}\left(-\rho_{12}\right)$ ] plotted as a function of probe detuning for three values of probe Rabi frequency $\Omega_{12}$. Both parameters are measured in units of $\Gamma_{21}$. The strength of the control laser is $\Omega_{23}=2 \pi \times 10 \mathrm{MHz}$. The left-hand side is for a closed system and the right-hand side is for an open system.

and the two variables

$$
\begin{aligned}
A & =\left[-\frac{1}{2} \frac{\Omega_{12}^{2}}{\Gamma_{2} \gamma_{12}}+\ldots\right], \\
\text { and } B & =\left[-\frac{1}{4} \frac{\Omega_{12}^{2}}{\gamma_{13} \gamma_{23}}+\frac{1}{16} \frac{\Omega_{12}^{4}}{\gamma_{13}^{2} \gamma_{23}^{2}}+\ldots\right],
\end{aligned}
$$

arise from the modified population differences. As expected, the expression for $\rho_{12}$ reduces to the weak-probe expression when $A=B=0$.

The numerical solutions of $\operatorname{Im}\left(-\rho_{12}\right)$ for three-level closed and open systems at three values of probe Rabi frequency $\left(\Omega_{12}\right)$ are shown in Fig. 3. The control laser is taken to have a strength of $\Omega_{23}=2 \pi \times 10 \mathrm{MHz}\left(1.7 \Gamma_{32}\right)$. To make the comparison reasonable for the two cases, the decay rate from $|3\rangle \rightarrow|2\rangle$ for the closed system is taken to be $\Gamma_{32}+\Gamma_{34}+\Gamma_{35}$, and the decay rate from $|3\rangle \rightarrow|4\rangle$ for the open system is taken to be $\Gamma_{34}+\Gamma_{35}$. This ensures that the decay term governing $\rho_{13}$ is the same in both cases. Both solutions are given at $t=100 \mu \mathrm{s}$.

Let us first consider the results for the closed system shown on the left-hand side. When $\Omega_{12}$ is very small (the weak-probe limit of $0.1 \Gamma_{21}$ ), we see the usual EIT dip at line center. When the power in increased to $2 \Gamma_{21}$, the EIT dip decreases, which can be interpreted as arising due to interference between new absorption paths created by the probe laser that were negligible when the laser was weak. This decrease continues with increased probe power so that, at $6 \Gamma_{21}$, the EIT dip almost completely disappears. For open systems, shown on the right- hand side of the figure, population loss into metastable levels is not significant in the weak-probe limit, as expected. When the probe power is increased, population loss causes the overall absorption amplitude to go down. However, competition between optical pumping and EIT allows the EIT dip to survive but with a split line shape. Such a splitting of the EIT line has also been observed before by us under strong-probe conditions in a closed ladder system, but in that case it arose due to thermal averaging [14]. At the highest power of $6 \Gamma_{21}$, population loss dominates and we see a wide dip at line center with 30,000 times less absorption.

We have also done simulations at other control-laser strengths. Apart from increased power broadening, the general features of the strong-probe modifications are the same.

\section{RESULTS IN A FOUR-LEVEL SYSTEM}

In a four-level system, there is enhanced absorption in the middle of the EIT dip at line center. This narrow feature, called Electromagnetically Induced Transparency and Absorption (EITA) [10], arises due to coherences created between levels $|1\rangle$ and $|4\rangle$. The coherences are generated by the simultaneous driving of transitions $|1\rangle \leftrightarrow|2\rangle,|2\rangle \leftrightarrow|3\rangle$, and $|3\rangle \leftrightarrow|4\rangle$ by three different lasers. As in the three-level case, all the population can be assumed to be in the ground level in the weak-probe limit, i.e., $\rho_{11}=1$, and $\rho_{22}=\rho_{33}=\rho_{44}=0$. This assumption breaks down when the probe is strong. Series expansion of $\rho_{12}$ in powers of $\Omega_{12}$ yields

$$
\rho_{12}=\frac{-\frac{i}{2} \frac{\Omega_{12}}{\gamma_{12}}\left[1-\frac{\Omega_{12}^{2}}{4 \Gamma_{12}}\left(\frac{1}{\gamma_{12}}+\frac{1}{\gamma_{12}^{*}}\right)+\ldots\right]}{1+\frac{\Omega_{23}^{2}}{4 \gamma_{12} \gamma_{13}} /\left(1+\frac{\Omega_{34}^{2}}{4 \gamma_{13} \gamma_{14}}\right)},
$$

where

$$
\begin{aligned}
& \gamma_{12}=\Gamma_{21} / 2+i \Delta_{12}, \\
& \gamma_{13}=\left(\Gamma_{32}+\Gamma_{34}+\Gamma_{35}\right) / 2+i\left(\Delta_{12}+\Delta_{23}\right), \\
& \gamma_{14}=\Gamma_{41} / 2+i\left(\Delta_{12}+\Delta_{23}-\Delta_{34}\right) .
\end{aligned}
$$

The numerical solutions of $\operatorname{Im}\left(-\rho_{12}\right)$ from the densitymatrix equations for both closed systems (with $\Gamma_{35}=0$ ) and open systems are shown in Fig. 4. The calculations are done with the control-laser strengths of $\Omega_{23}=2 \pi \times$ $10 \mathrm{MHz}\left(1.7 \Gamma_{32}\right)$ and $\Omega_{34}=5 \mathrm{MHz}\left(3.2 \Gamma_{34}\right)$. As in the case of the three-level system, the decay term governing $\rho_{13}$ is made same by taking the decay rate from $|3\rangle \rightarrow|4\rangle$ for the closed system to be $\Gamma_{34}+\Gamma_{35}$. The solutions are shown at $t=500 \mu \mathrm{s}$.

In closed systems (shown on the left-hand side of the figure), the phenomenon of enhanced absorption seen in the weak-probe limit of $\Omega_{12}=0.1 \Gamma_{21}$ becomes less prominent when the Rabi frequency is increased to $2 \Gamma_{21}$. The linewidth of the feature also increases. The feature inverts to an enhanced transmission dip when the Rabi 


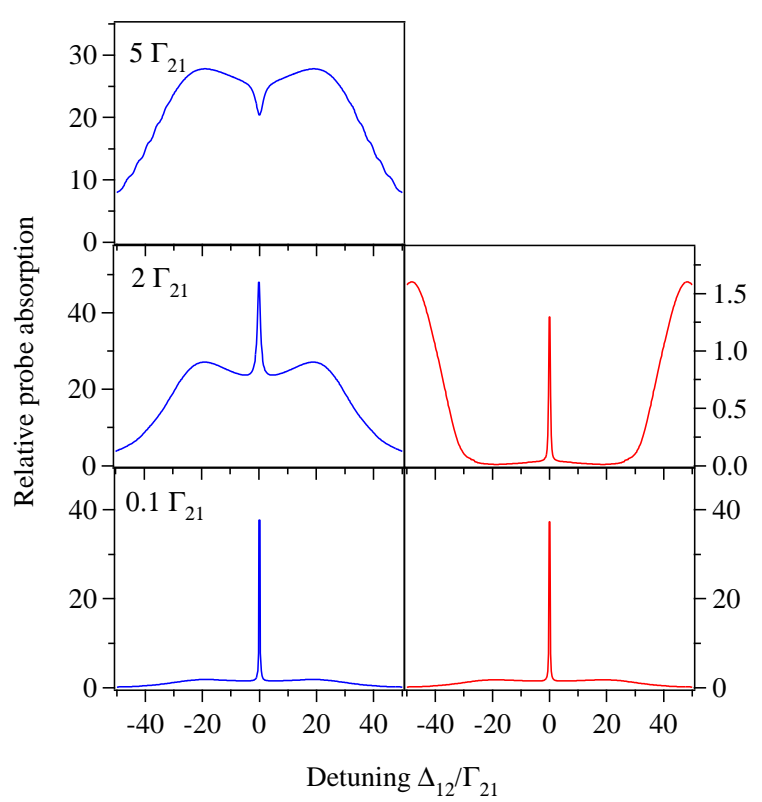

FIG. 4: Relative probe absorption [defined as $\operatorname{Im}\left(-\rho_{12}\right)$ ] plotted as a function of probe detuning for different values of probe Rabi frequency $\Omega_{12}$. Both parameters are measured in units of $\Gamma_{21}$. The two control lasers have strengths of $\Omega_{23}=2 \pi \times 10 \mathrm{MHz}$ and $\Omega_{34}=2 \pi \times 5 \mathrm{MHz}$. The left-hand side is for a closed system and the right-hand side is for an open system. The absorption at $\Omega_{12}=5 \Gamma_{12}$ for an open system is negligible, and not shown.

frequency becomes $5 \Gamma_{21}$. In open systems (shown on the right-hand side of the figure), the line shape is similar to that of the closed system in the weak-probe limit, as expected. However, when the probe Rabi frequency is increased to $2 \Gamma_{21}$, optical pumping into the metastable level causes population loss and reduces the absorption by a factor of 30 . But the overall EITA feature survives with the same linewidth. At very high powers, population loss makes the absorption disappear completely and is not shown.

\section{CONCLUSIONS}

In conclusion, we have seen that it is important to consider the effects of a strong probe laser in several applications of the phenomenon of EIT. The strong probe laser causes population transfer and creates new pathways for probe absorption. We solve the set of density-matrix equations numerically to understand these effects in both closed and open systems. For a closed three-level ladder system, the EIT dip at line center disappears as the probe laser becomes strong. In an open system, the absorption level goes down because of population loss but the general EIT feature at line center survives. For a four-level system, there is a narrow enhanced-absorption peak at line center. This converts to an EIT dip under strongprobe conditions in a closed system. However, in an open system, the enhanced-absorption feature survives even at high probe powers albeit at a reduced absorption level. These differences should be important in proposed clock applications in four-level systems.

\section{Acknowledgments}

This work was supported by the Department of Science and Technology, India. V.N. acknowledges support from the Homi Bhabha Fellowship Council and K.P. from the Council of Scientific and Industrial Research, India.
[1] A. Imamoğlu and S. E. Harris, Opt. Lett. 14, 1344 (1989), URL http://ol.osa.org/abstract.cfm?URI=ol-14-24-1344

[2] G. S. Agarwal, Phys. Rev. Lett. 67, 980 (1991).

[3] U. D. Rapol and V. Natarajan, Europhys. Lett. 60, 195 (2002).

[4] A. Krishna, K. Pandey, A. Wasan, and V. Natarajan, Europhys. Lett. 72, 221 (2005).

[5] S. E. Harris, J. E. Field, and A. Imamoğlu, Phys. Rev. Lett. 64, 1107 (1990).

[6] Y. Zhang, U. Khadka, B. Anderson, and M. Xiao, Physical Review Letters 102, 013601 (pages 4) (2009), URL http://link.aps.org/abstract/PRL/v102/e013601.

[7] S. Wielandy and A. L. Gaeta, Phys. Rev. Lett. 81, 3359 (1998).

[8] K. Pandey, A. Wasan, and V. Natarajan, J. Phys. B 41, 225503 (8pp) (2008), URL http://stacks.iop.org/0953-4075/41/225503
[9] L. V. Hau, S. E. Harris, Z. Dutton, and C. H. Behroozi, Nature (London) 397, 594 (1999).

[10] T. Hong, C. Cramer, W. Nagourney, and E. N. Fortson, Phys. Rev. Lett. 94, 050801 (2005).

[11] R. Santra, E. Arimondo, T. Ido, C. H. Greene, and J. Ye, Phys. Rev. Lett. 94, 173002 (2005).

[12] A. V. Taichenachev, V. I. Yudin, C. W. Oates, C. W. Hoyt, Z. W. Barber, and L. Hollberg, Phys. Rev. Lett. 96, 083001 (2006).

[13] S. Wielandy and A. L. Gaeta, Phys. Rev. A 58, 2500 (1998).

[14] K. Pandey and V. Natarajan, J. Phys. B 41, 185504 (4pp) (2008), URL http://stacks.iop.org/0953-4075/41/185504.

[15] T. Loftus, J. R. Bochinski, and T. W. Mossberg, Phys. Rev. A 66, 013411 (2002). 